\title{
Neutrino interaction with matter in a noninertial frame
}

\section{Maxim Dvornikov}

Institute of Physics, University of São Paulo, CP 66318, CEP 05315-970 São Paulo, SP, Brazil

Pushkov Institute of Terrestrial Magnetism, Ionosphere and Radiowave Propagation (IZMIRAN), 142190 Troitsk, Moscow, Russia

Nonlinear Physics Centre, Research School of Physics and Engineering, Australian National University, 2601 Canberra, ACT, Australia

Physics Department, Tomsk State University, 634050 Tomsk, Russia

E-mail: maxim.dvornikov@usp.br

ABSTRACT: We study the system of massive and mixed neutrinos interacting with background matter moving with an acceleration. We start with the derivation of the Dirac equation for a single neutrino in the noninertial frame where matter is at rest. A particular case of matter rotating with a constant angular velocity is considered. The Dirac equation is solved and the neutrino energy levels are found for ultrarelativistic particles propagating in rotating matter. Then we generalize our results to include several neutrino generations and consider mixing between them. Using the relativistic quantum mechanics approach we derive the effective Schrödinger equation for the description of neutrino flavor oscillations in rotating matter. We obtain the resonance condition for neutrino oscillations and examine how it can be affected by the matter rotation. We also compare our results with the findings of other authors who studied analogous problem previously.

Keywords: Neutrino Physics, Classical Theories of Gravity, Integrable Equations in Physics

ARXIV EPRINT: 1408.2735 


\section{Contents}

1 Introduction $\quad 1$

2 Neutrino interaction with background matter 2

3 Massive neutrinos in noninertial frames 3

3.1 Neutrino motion in a rotating frame 4

4 Flavor oscillations of Dirac neutrinos in rotating matter 9

5 Summary and discussion $\quad 11$

\section{Introduction}

Nowadays it is commonly believed that neutrinos are massive particles and there is a nonzero mixing between different neutrino generations. These neutrino properties result in transitions between neutrino flavors, or neutrino oscillations [1]. It is also known that various external fields, like electroweak interaction of neutrinos with background fermions [2], neutrino electromagnetic interaction [3], and neutrino interaction with a strong gravitational field [4], can also influence the process of neutrino oscillations.

As shown in ref. [5], noninertial effects in accelerated and rotating frames can affect neutrino propagation and oscillations. The consideration of the reference frame rotation is particularly important for astrophysical neutrinos emitted by a rapidly rotating compact star, e.g., a pulsar. For example, the possibility of the pulsar spin down by the neutrino emission and interaction with rotating matter was recently discussed in ref. [6]. It should be noted that besides elementary particle physics, various processes in noninertial frames are actively studied in condensed matter physics. For instance, the enhancement of the spin current in a semiconductor moving with an acceleration was recently predicted in ref. [7].

In the present work we shall study the neutrino interaction with background matter in a rotating frame. We assume that neutrinos can interact with background fermions by means of electroweak forces. We also take that neutrino mass eigenstates are Dirac particles. In our treatment we account for the noninertial effects since we find the exact solution of a Dirac equation for a neutrino moving in the curved space-time with a metric corresponding a rotating frame.

This work is organized in the following way. In section 2 , we start with the brief description of the neutrino interaction with background matter in Minkowski space-time. We consider matter moving with a constant velocity and discuss both neutrino flavor and mass eigenstates. Then, in section 3, we study background matter moving with an acceleration. The Dirac equation, including noninertial effects, for a neutrino interacting 
with such matter is written down in a frame where matter is at rest. In section 3.1, we solve the Dirac equation and find the neutrino energy spectrum for ultrarelativistic neutrinos moving in matter rotating with a constant angular velocity. Then, in section 4 , we apply our results for the description of neutrino oscillations in rotating background matter. The effective Schrödinger equation governing neutrino oscillations is derived and the new resonance condition is obtained. We consider how the matter rotation can affect the resonance in neutrino oscillation in a realistic astrophysical situation. Finally, in section 5, we summarize our results and compare them with the previous findings of other authors.

\section{Neutrino interaction with background matter}

In this section we describe the interaction of different neutrino flavors with background matter in a flat space-time. We discuss a general case of matter moving with a constant mean velocity and having a mean polarization. Then we consider the matter interaction of neutrino mass eigenstates, which are supposed to be Dirac particles.

The interaction of the neutrino flavor eigenstates $\nu_{\alpha}, \alpha=e, \mu, \tau$, with background matter in the flat space-time is described by the following effective Lagrangian [8]:

$$
\mathcal{L}_{\text {eff }}=-\sum_{\alpha} \bar{\nu}_{\alpha} \gamma_{\mu}^{\mathrm{L}} \nu_{\alpha} \cdot f_{\alpha}^{\mu},
$$

where $\gamma_{\mu}^{\mathrm{L}}=\gamma_{\mu}\left(1-\gamma^{5}\right) / 2, \gamma^{\mu}=\left(\gamma^{0}, \gamma\right)$ are the Dirac matrices, and $\gamma^{5}=\mathrm{i} \gamma^{0} \gamma^{1} \gamma^{2} \gamma^{3}$.

The interaction Lagrangian in eq. (2.1) is derived in the mean field approximation using the effective external currents $f_{\alpha}^{\mu}$ depending on the characteristics of background matter as [9]

$$
f_{\alpha}^{\mu}=\sqrt{2} G_{\mathrm{F}} \sum_{f}\left(q_{\alpha, f}^{(1)} j_{f}^{\mu}+q_{\alpha, f}^{(2)} \lambda_{f}^{\mu}\right)
$$

where $G_{\mathrm{F}}$ is the Fermi constant and the sum is taken over all background fermions $f$. Here

$$
j_{f}^{\mu}=n_{f} u_{f}^{\mu},
$$

is the hydrodynamic current and

$$
\lambda_{f}^{\mu}=n_{f}\left(\left(\boldsymbol{\zeta}_{f} \mathbf{u}_{f}\right), \boldsymbol{\zeta}_{f}+\frac{\mathbf{u}_{f}\left(\boldsymbol{\zeta}_{f} \mathbf{u}_{f}\right)}{1+u_{f}^{0}}\right),
$$

is the four vector of the matter polarization. In eqs. (2.3) and (2.4), $n_{f}$ is the invariant number density (the density in the rest frame of fermions), $\boldsymbol{\zeta}_{f}$ is the invariant polarization (the polarization in the rest frame of fermions), and $u_{f}^{\mu}=\left(u_{f}^{0}, \mathbf{u}_{f}\right)$ is the four velocity. To derive eqs. (2.2)-(2.4) it is crucial that background fermions have constant velocity. Only in this situation one can make a boost to the rest frame of the fermions where $n_{f}$ and $\boldsymbol{\zeta}_{f}$ are defined.

The coefficients $q_{\alpha, f}^{(1,2)}$ in eq. (2.2) can be found in the explicit form if we discuss the system of active neutrinos moving in matter composed of electrons $e$, protons $p$, and neutrons $n$. In this situation, one gets for $\nu_{e}[9]$,

$$
q_{\nu_{e}, f}^{(1)}=I_{\mathrm{L} 3}^{(f)}-2 Q_{f} \sin ^{2} \theta_{\mathrm{W}}+\delta_{e f}, \quad q_{\nu_{e}, f}^{(2)}=-I_{\mathrm{L} 3}^{(f)}-\delta_{e f},
$$


where $I_{\mathrm{L} 3}^{(f)}$ is the third component of the weak isospin of type $f$ fermions, $Q_{f}$ is the value of their electric charge, $\theta_{\mathrm{W}}$ is the Weinberg angle, $\delta_{e f}=1$ for electrons and vanishes for protons and neutrons. To get these coefficients for $\nu_{\mu, \tau}$ the symbol $\delta_{e f}$ should be eliminated from eq. (2.5).

It was experimentally confirmed (see, e.g., ref. [10]) that the flavor neutrino eigenstates are the superposition of the neutrino mass eigenstates, $\psi_{i}, i=1,2, \ldots$,

$$
\nu_{\alpha}=\sum_{i} U_{\alpha i} \psi_{i}
$$

where $\left(U_{\alpha i}\right)$ is the unitary mixing matrix. The transformation in eq. (2.6) diagonalizes the neutrino mass matrix. Only using the neutrino mass eigenstates we can reveal the nature of neutrinos, i.e. say whether they are Dirac or Majorana particles. Despite the great experimental efforts to shed light upon the nature of neutrinos [11], this issue still remains open. Here we shall suppose that $\psi_{i}$ correspond to Dirac fields.

The effective Lagrangian for the interaction of $\psi_{i}$ with background matter can be obtained using eqs. (2.1) and (2.6),

$$
\mathcal{L}_{\text {eff }}=-\sum_{i j} \bar{\psi}_{i} \gamma_{\mu}^{\mathrm{L}} \psi_{j} \cdot g_{i j}^{\mu}
$$

where

$$
g_{i j}^{\mu}=\sum_{\alpha} U_{\alpha i}^{*} U_{\alpha j} f_{\alpha}^{\mu}
$$

is the nondiagonal effective potential in the mass eigenstates basis.

Using eq. (2.7) one obtains that the corresponding Dirac equations for the neutrino mass eigenstates are coupled,

$$
\left[\mathrm{i} \gamma^{\mu} \partial_{\mu}-m_{i}-\gamma_{\mu}^{\mathrm{L}} g_{i i}^{\mu}\right] \psi_{i}=\sum_{j \neq i} \gamma_{\mu}^{\mathrm{L}} g_{i j}^{\mu} \psi_{j}
$$

where $m_{i}$ is the mass of $\psi_{i}$. One can proceed in the analytical analysis of eq. (2.9) if we exactly account for only the diagonal effective potentials $g_{i i}^{\mu}$. To take into account the r.h.s. of eq. (2.9), depending on the nondiagonal elements of the matrix $\left(g_{i j}^{\mu}\right)$, with $i \neq j$, one should apply a perturbative method (see section 4 below).

\section{Massive neutrinos in noninertial frames}

In this section we generalize the Dirac equation for a neutrino interacting with a background matter to the situation when the velocity of the matter motion is not constant. In particular, we study the case of the matter rotation with a constant angular velocity. Then we obtain the solution of the Dirac equation and find the energy spectrum.

If we discuss a neutrino mass eigenstate propagating in a nonuniformly moving matter, the expressions for $f_{\alpha}^{\mu}$ in eqs. (2.2)-(2.4) become invalid since they are derived under the assumption of the unbroken Lorentz invariance. The most straightforward way to describe the neutrino evolution in matter moving with an acceleration is to rewrite the 
Dirac equation for a neutrino in the noninertial frame where matter is at rest. In this case one can unambiguously define the components of $f_{\alpha}^{\mu}$. Assuming that background fermions are unpolarized, we find that in this reference frame

$$
f_{\alpha}^{0}=\sqrt{2} G_{\mathrm{F}} \sum_{f} q_{\alpha, f}^{(1)} n_{f} \neq 0,
$$

with the rest of the effective potentials being equal to zero.

It is known that the motion of a test particle in a noninertial frame is equivalent to the interaction of this particle with a gravitational field. The Dirac equation for a massive neutrino moving in a curved space-time and interacting with background matter can be obtained by the generalization of eq. (2.9) (see also ref. [12]),

$$
\left[\mathrm{i} \gamma^{\mu}(x) \nabla_{\mu}-m\right] \psi=\frac{1}{2} \gamma_{\mu}(x) g^{\mu}\left[1-\gamma^{5}(x)\right] \psi,
$$

where $\gamma_{\mu}(x)$ are the coordinate dependent Dirac matrices, $\nabla_{\mu}=\partial_{\mu}+\Gamma_{\mu}$ is the covariant derivative, $\Gamma_{\mu}$ is the spin connection, $\gamma^{5}(x)=-\frac{i}{4 !} E^{\mu \nu \alpha \beta} \gamma_{\mu}(x) \gamma_{\nu}(x) \gamma_{\alpha}(x) \gamma_{\beta}(x), E^{\mu \nu \alpha \beta}=$ $\frac{1}{\sqrt{-g}} \varepsilon^{\mu \nu \alpha \beta}$ is the covariant antisymmetric tensor in curved space-time, $g_{\mu \nu}$ is the metric tensor, and $g=\operatorname{det}\left(g_{\mu \nu}\right)$. Note that in eq. (3.2) we account for only the diagonal neutrino interaction with matter. That is why we omit the index $i$ in order not to encumber the notation: $m \equiv m_{i}$ etc. It should be noted that analogous Dirac equation was discussed in ref. [13].

We shall be interested in the neutrino motion in matter rotating with the constant angular velocity $\omega$. Choosing the corotating frame we get that only $g^{0} \equiv g_{i i}^{0}$ is nonvanishing, cf. eqs. (2.8) and (3.1).

\subsection{Neutrino motion in a rotating frame}

The interval in the rotating frame is [14]

$$
\mathrm{d} s^{2}=g_{\mu \nu} \mathrm{d} x^{\mu} \mathrm{d} x^{\nu}=\left(1-\omega^{2} r^{2}\right) \mathrm{d} t^{2}-\mathrm{d} r^{2}-2 \omega r^{2} \mathrm{~d} t \mathrm{~d} \phi-r^{2} \mathrm{~d} \phi^{2}-\mathrm{d} z^{2},
$$

where we use the cylindrical coordinates $x^{\mu}=(t, r, \phi, z)$. One can check that the metric tensor in eq. (3.3) can be diagonalized, $\eta_{a b}=e_{a}{ }^{\mu} e_{b}^{\nu} g_{\mu \nu}$, if we use the following vierbein vectors:

$$
\begin{aligned}
e_{0}^{\mu} & =\left(\frac{1}{\sqrt{1-\omega^{2} r^{2}}}, 0,0,0\right), \\
e_{1}^{\mu} & =(0,1,0,0), \\
e_{2}^{\mu} & =\left(\frac{\omega r}{\sqrt{1-\omega^{2} r^{2}}}, 0, \frac{\sqrt{1-\omega^{2} r^{2}}}{r}, 0\right), \\
e_{3}^{\mu} & =(0,0,0,1) .
\end{aligned}
$$

Here $\eta_{a b}=\operatorname{diag}(1,-1,-1,-1)$ is the metric in a locally Minkowskian frame.

Let us introduce the Dirac matrices in a locally Minkowskian frame by $\gamma^{\bar{a}}=e_{\mu}^{a} \gamma^{\mu}(x)$, where $e^{a}{ }_{\mu}$ is the inverse vierbein: $e^{a}{ }_{\mu} e_{b}^{\mu}=\delta_{b}^{a}$. Starting from now, we shall mark an index 
with a bar to show that a gamma matrix is defined in a locally Minkowskian frame. We notice that $g=-\left[\operatorname{det}\left(e^{a}{ }_{\mu}\right)\right]^{2}$ and $E^{\mu \nu \alpha \beta} e_{\mu}^{m} e^{n}{ }_{\nu} e_{a}^{\alpha} e^{\beta}{ }_{b}=\varepsilon^{m n a b}$. Thus $\gamma^{5}(x)=\mathrm{i} \gamma^{\overline{0}} \gamma^{\overline{1}} \gamma^{\overline{2}} \gamma^{\overline{3}}=$ $\gamma^{\overline{5}}$ does not depend on coordinates.

The spin connection has the form [12]

$$
\Gamma_{\mu}=-\frac{\mathrm{i}}{4} \sigma^{a b} \omega_{a b \mu}, \quad \omega_{a b \mu}=e_{a}^{\nu} e_{b \nu ; \mu},
$$

where $\sigma_{a b}=\frac{\mathrm{i}}{2}\left[\gamma_{\bar{a}}, \gamma_{\bar{b}}\right]_{-}$are the generators of the Lorentz transformations in a locally Minkowskian frame. Here the semicolon stays for the covariant derivative: $A_{\nu ; \mu}=\partial_{\mu} A_{\nu}-$ $\Gamma_{\mu \nu}^{\alpha} A_{\alpha}$, with $\Gamma_{\mu \nu}^{\alpha}$ being the Christoffel symbol of the second kind. The components of the connection one-form $\omega_{a b}=\omega_{a b \mu} \mathrm{d} x^{\mu}$ are

$$
\begin{aligned}
& \omega_{01 \mu}=-\omega_{10 \mu}=\left(-\frac{\omega^{2} r}{\sqrt{1-\omega^{2} r^{2}}}, 0,-\frac{\omega r}{\sqrt{1-\omega^{2} r^{2}}}, 0\right), \\
& \omega_{02 \mu}=-\omega_{20 \mu}=\left(0, \frac{\omega}{1-\omega^{2} r^{2}}, 0,0\right), \\
& \omega_{12 \mu}=-\omega_{21 \mu}=\left(\frac{\omega}{\sqrt{1-\omega^{2} r^{2}}}, 0, \frac{1}{\sqrt{1-\omega^{2} r^{2}}}, 0\right),
\end{aligned}
$$

with the rest of the components being equal to zero.

Using eqs. (3.4)-(3.6), the Dirac eq. (3.2) can be rewritten as

$$
\begin{aligned}
{[\mathcal{D}-m] \psi=\frac{1}{2} \sqrt{1-\omega^{2} r^{2}} \gamma^{\overline{0}} g^{0}\left(1-\gamma^{\overline{5}}\right) \psi } \\
\mathcal{D}=\mathrm{i} \frac{\gamma^{\overline{0}}+\omega r \gamma^{\overline{2}}}{\sqrt{1-\omega^{2} r^{2}}} \partial_{0}+\mathrm{i} \gamma^{\overline{1}}\left(\partial_{r}+\frac{1}{2 r}\right)+\mathrm{i} \gamma^{\overline{2}} \frac{\sqrt{1-\omega^{2} r^{2}}}{r} \partial_{\phi}+\mathrm{i} \gamma^{\overline{3}} \partial_{z} \\
\quad-\frac{\omega}{2\left(1-\omega^{2} r^{2}\right)} \gamma^{\overline{3}} \gamma^{\overline{5}} .
\end{aligned}
$$

The analogous Dirac equation was recently derived in ref. [15]. Since eq. (3.7) does not explicitly contain $t, \phi$, and $z$, its solution can be expressed as

$$
\psi=\exp \left(-\mathrm{i} E t+\mathrm{i} J_{z} \phi+\mathrm{i} p_{z} z\right) \psi_{r},
$$

where $\psi_{r}=\psi_{r}(r)$ is the spinor depending on the radial coordinate, $J_{z}=\frac{1}{2}-l$ (see, e.g., ref. [16]), and $l=0, \pm 1, \pm 2, \ldots$.

In eq. (3.7) one can neglect terms $\sim(\omega r)^{2}$. Indeed, if we study a neutrino in a rotating pulsar, then $r \lesssim 10 \mathrm{~km}$ and $\omega \lesssim 10^{3} \mathrm{~s}^{-1}$. Thus $(\omega r)^{2} \lesssim 1.1 \times 10^{-3}$ is a small parameter. Therefore eq. (3.7) can be transformed to

$$
\begin{aligned}
{\left[\mathrm{i} \gamma^{\overline{1}}\left(\partial_{r}+\frac{1}{2 r}\right)-\gamma^{\overline{2}}\left(\frac{J_{z}}{r}-\omega r E\right)+\gamma^{\overline{0}}\left(E-\frac{g^{0}}{2}\right)\right.} & -\gamma^{\overline{3}} p_{z} \\
& \left.+\frac{g^{0}}{2} \gamma^{\overline{0}} \gamma^{\overline{5}}-\frac{\omega}{2} \gamma^{\overline{3}} \gamma^{\overline{5}}-m\right] \psi_{r}=0
\end{aligned}
$$

where we keep only the terms linear in $\omega$. It should be noted that the term $\sim \omega \gamma^{\overline{3}} \gamma^{\overline{5}}$ in eq. (3.9) is equivalent to the neutrino interaction with matter moving with an effective velocity. 
Let us transform the wave function as

$$
\psi_{r}=U_{3} \tilde{\psi}_{r}, \quad U_{3}=\exp \left(\frac{v}{2} \gamma^{\overline{0}} \gamma^{\overline{3}}\right)=\cosh \frac{v}{2}+\gamma^{\overline{0}} \gamma^{\overline{3}} \sinh \frac{v}{2},
$$

where $v$ is a real parameter satisfying $\tanh v=\omega / g^{0}$. Such a transformation is equivalent to a boost. If we again study a neutrino moving inside a rotating pulsar, then $g^{0} \sim$ $G_{\mathrm{F}} n_{n} \sim 10 \mathrm{eV}$, where $n_{n} \sim 10^{38} \mathrm{~cm}^{-3}$ is the neutron density. Thus $v \sim 10^{-13} \ll 1$ and the transformation in eq. (3.10) exists. The spinor $\tilde{\psi}_{r}$ obeys the equation,

$$
\left[\gamma^{\bar{a}} Q_{a}+\frac{\tilde{g}^{0}}{2} \gamma^{\overline{0}} \gamma^{\overline{5}}-m\right] \tilde{\psi}_{r}=0
$$

where $Q^{a}=\left(\tilde{E},-\mathrm{i}\left(\partial_{r}+\frac{1}{2 r}\right),\left(\frac{J_{z}}{r}-\omega r E\right), \tilde{p}_{z}\right)$. It is convenient to represent $Q^{a}=q^{a}-$ $q_{\text {eff }} A_{\text {eff }}^{a}$, where $q^{a}=\left(\tilde{E},-\mathrm{i} \partial_{r}, 0, p_{z}\right), A_{\text {eff }}^{a}=\left(0, \frac{\mathrm{i}}{2 q_{\text {eff }} r}, \frac{1}{q_{\text {eff }}}\left(\omega r E-\frac{J_{z}}{r}\right), 0\right)$ is the vector potential of the effective electromagnetic field, and $q_{\text {eff }}$ is the effective electric charge. Here

$$
\begin{aligned}
\tilde{E} & =E^{\prime} \cosh v-p_{z} \sinh v, \\
\tilde{p}_{z} & =p_{z} \cosh v-E^{\prime} \sinh v, \\
\tilde{g}^{0} & =g^{0} \cosh v-\omega \sinh v,
\end{aligned}
$$

and $E^{\prime}=E-\frac{g^{0}}{2}$.

We shall look for the solution of eq. (3.11) as

$$
\tilde{\psi}_{r}=\Pi \Phi, \quad \Pi=\gamma^{\bar{a}} Q_{a}-\frac{\tilde{g}^{0}}{2} \gamma^{\overline{0}} \gamma^{\overline{5}}+m
$$

where $\Phi=\Phi(r)$ is a new spinor. This spinor obeys the equation,

$$
\begin{aligned}
{\left[\partial_{r}^{2}+\frac{\partial_{r}}{r}-\frac{1}{4 r^{2}}+\tilde{E}^{2}-\tilde{p}_{z}^{2}-\left(\frac{J_{z}}{r}-\omega r E\right)^{2}-m^{2}+\frac{\left(\tilde{g}^{0}\right)^{2}}{4}\right.} & \\
& \left.+\left(E \omega+\frac{J_{z}}{r^{2}}\right) \Sigma_{3}-\tilde{g} \tilde{g}^{0} \gamma^{\overline{5}}+m \tilde{g}^{0} \gamma^{\overline{0}} \gamma^{\overline{5}}\right] \Phi=0
\end{aligned}
$$

which can be obtained using eq. (3.11).

Eq. (3.14) can be solved for ultrarelativistic neutrinos when the particle mass is neglected. In this case we represent $\Phi=v \varphi$, where $v$ is a constant spinor and $\varphi=\varphi(r)$ is a scalar function. We notice that both $\Sigma_{3}=\gamma^{\overline{0}} \gamma^{\overline{3}} \gamma^{\overline{5}}$ and $\gamma^{\overline{5}}$ commute with the operator of eq. (3.14) when $m \rightarrow 0$. Taking into account that $\left[\Sigma_{3}, \gamma^{\overline{5}}\right]_{-}=0$, one gets that the spinor $v$ satisfies $\Sigma_{3} v=\sigma v$ and $\gamma^{\overline{5}} v=\chi v$, where $\sigma= \pm 1$ and $\chi= \pm 1$.

We can choose two linearly independent spinors $v$ as

$$
v_{-}=v(\chi=+1, \sigma=-1)=\left(\begin{array}{l}
0 \\
1 \\
0 \\
0
\end{array}\right), \quad v_{+}=v(\chi=-1, \sigma=+1)=\left(\begin{array}{l}
0 \\
0 \\
1 \\
0
\end{array}\right) .
$$


To derive eq. (3.15) we use the Dirac matrices in form,

$$
\gamma^{\overline{0}}=\left(\begin{array}{cc}
0 & -1 \\
-1 & 0
\end{array}\right), \quad \gamma^{\bar{k}}=\left(\begin{array}{cc}
0 & \sigma_{k} \\
-\sigma_{k} & 0
\end{array}\right), \quad \gamma^{\overline{5}}=\left(\begin{array}{cc}
1 & 0 \\
0 & -1
\end{array}\right),
$$

that corresponds to the chiral representation [17]. In eq. (3.16) $\sigma_{k}$ are the Pauli matrices.

Introducing the new variable $\rho=E \omega r^{2}$, we get the equation for $\varphi_{\sigma}$,

$$
\left[\rho \frac{\mathrm{d}^{2}}{\mathrm{~d} \rho}+\frac{\mathrm{d}}{\mathrm{d} \rho}-\frac{1}{4 \rho}\left(l+\frac{\sigma-1}{2}\right)^{2}-\frac{\rho}{4}-\frac{1}{2}\left(l-\frac{\sigma+1}{2}\right)+\kappa\right] \varphi_{\sigma}=0
$$

where

$$
\kappa=\frac{1}{4 E \omega}\left(\tilde{E}^{2}-\tilde{p}_{z}^{2}+\frac{\left(\tilde{g}^{0}\right)^{2}}{4}-\tilde{g}^{0} \tilde{E} \chi\right) .
$$

The solution of eq. (3.17) which vanishes at the infinity, $\varphi_{\sigma}(\rho \rightarrow \infty) \rightarrow 0$, can be expressed as $\varphi_{+}=I_{N, s}$ and $\varphi_{-}=I_{N-1, s}$, where $N=0,1,2, \ldots, s=N-l$, and $I_{N, s}=I_{N, s}(\rho)$ is the Laguerre function,

$$
I_{N, s}(\rho)=\sqrt{\frac{s !}{N !}} \exp \left(-\frac{\rho}{2}\right) \rho^{\frac{N-s}{2}} L_{s}^{N-s}(\rho) .
$$

Here $L_{s}^{\alpha}(\rho)$ are the associated Laguerre polynomials.

The energy spectrum can be obtained if we notice that $\kappa=N$ in eq. (3.18). Thus we get

$$
\begin{aligned}
{\left[E_{A}-2 N \omega-g^{0}\right]^{2} } & =(2 N \omega)^{2}+4 N \omega g^{0}+\left(p_{z}-\frac{\omega}{2}\right)^{2}, \\
{\left[E_{S}-2 N \omega\right]^{2} } & =(2 N \omega)^{2}+\left(p_{z}+\frac{\omega}{2}\right)^{2},
\end{aligned}
$$

where $E_{A}=E(\chi=+1)$ and $E_{S}=E(\chi=-1)$ are the energies of active and sterile neutrinos respectively. Comparing the expression for $E_{S} \approx 2 N \omega+\sqrt{(2 N \omega)^{2}+p_{z}^{2}}$ with the energy of a neutrino in an inertial nonrotating frame $\sqrt{\mathbf{p}_{\perp}^{2}+p_{z}^{2}}$, where $\mathbf{p}_{\perp}$ is the momentum in the equatorial plane, we can identify $2 N \omega$ inside the square root as $\left|\mathbf{p}_{\perp}\right|$. It should be also noted that the term $2 N \omega$, which additively enters to both $E_{A}$ and $E_{S}$, is due to the noninertial effects for a Dirac fermion in a rotating frame [18].

Using eqs. (3.10), (3.13), (3.15), and (3.20) we can get the wave function $\psi_{r}$ in the explicit form,

$$
\psi_{r}^{\mathrm{L}}=U_{3} \Pi v_{-} \varphi_{-}=\left(\begin{array}{c}
0 \\
\eta
\end{array}\right), \quad \psi_{r}^{\mathrm{R}}=U_{3} \Pi v_{+} \varphi_{+}=\left(\begin{array}{c}
\xi \\
0
\end{array}\right)
$$

where

$$
\eta=\left(\begin{array}{c}
-\mathrm{i} C_{1} I_{N, s} \\
C_{2} I_{N-1, s}
\end{array}\right), \quad \xi=\left(\begin{array}{c}
C_{3} I_{N, s} \\
-\mathrm{i} C_{4} I_{N-1, s}
\end{array}\right)
$$


and

$$
\begin{aligned}
& C_{1}^{2}=\frac{E_{A} \omega}{\pi} \frac{\left(1-\omega / g^{0}\right)^{3 / 2}}{\left(1+\omega / g^{0}\right)^{1 / 2}} \frac{4 N \omega E_{A}}{\left(\tilde{E}_{A}+\tilde{p}_{z}-\tilde{g}^{0} / 2\right)^{2}\left(1+\omega / g^{0}\right)+4 N \omega E_{A}\left(1-\omega / g^{0}\right)}, \\
& C_{2}^{2}=\frac{E_{A} \omega}{\pi} \frac{\left(1+\omega / g^{0}\right)^{3 / 2}}{\left(1-\omega / g^{0}\right)^{1 / 2}} \frac{\left(\tilde{E}_{A}+\tilde{p}_{z}-\tilde{g}^{0} / 2\right)^{2}}{\left(\tilde{E}_{A}+\tilde{p}_{z}-\tilde{g}^{0} / 2\right)^{2}\left(1+\omega / g^{0}\right)+4 N \omega E_{A}\left(1-\omega / g^{0}\right)}, \\
& C_{3}^{2}=\frac{E_{S} \omega}{\pi} \frac{\left(1+\omega / g^{0}\right)^{3 / 2}}{\left(1-\omega / g^{0}\right)^{1 / 2}} \frac{\left(\tilde{E}_{S}+\tilde{p}_{z}+\tilde{g}^{0} / 2\right)^{2}}{\left(\tilde{E}_{S}+\tilde{p}_{z}+\tilde{g}^{0} / 2\right)^{2}\left(1+\omega / g^{0}\right)+4 N \omega E_{S}\left(1-\omega / g^{0}\right)}, \\
& C_{4}^{2}=\frac{E_{S} \omega}{\pi} \frac{\left(1-\omega / g^{0}\right)^{3 / 2}}{\left(1+\omega / g^{0}\right)^{1 / 2}} \frac{4 N \omega E_{S}}{\left(\tilde{E}_{S}+\tilde{p}_{z}+\tilde{g}^{0} / 2\right)^{2}\left(1+\omega / g^{0}\right)+4 N \omega E_{S}\left(1-\omega / g^{0}\right)} .
\end{aligned}
$$

To derive eqs. (3.21)-(3.23) we use the following properties of the Laguerre functions:

$$
\begin{aligned}
\left(\partial_{r}-\frac{l-1}{r}-\omega E r\right) I_{N-1, s}\left(\omega E r^{2}\right) & =-2 \sqrt{N \omega E} I_{N, s}\left(\omega E r^{2}\right), \\
\left(\partial_{r}+\frac{l}{r}+\omega E r\right) I_{N, s}\left(\omega E r^{2}\right) & =2 \sqrt{N \omega E} I_{N-1, s}\left(\omega E r^{2}\right),
\end{aligned}
$$

which can be verified using eq. (3.19).

In the following we shall study the important case when $\omega \ll g^{0}$. In this situation we get for the coefficients $C_{i}$,

$$
\begin{aligned}
& C_{1}^{2} \approx \frac{E_{A} \omega}{2 \pi} \frac{E_{A}-p_{z}-g^{0}}{E_{A}-g^{0}}, \quad C_{3}^{2} \approx \frac{\omega}{2 \pi}\left(E_{S}+p_{z}\right), \\
& C_{2}^{2} \approx \frac{E_{A} \omega}{2 \pi} \frac{E_{A}+p_{z}-g^{0}}{E_{A}-g^{0}}, \quad C_{4}^{2} \approx \frac{\omega}{2 \pi}\left(E_{S}-p_{z}\right),
\end{aligned}
$$

which result from eq. (3.23).

The solution given in eqs. (3.22)-(3.25) is valid for $N>0$. If $N=0$, the spinors $\eta$ and $\xi$ take the form

$$
\begin{aligned}
\eta & =\mathcal{N}_{A}\left(\begin{array}{c}
I_{0,0} \\
0
\end{array}\right), \quad \xi=\mathcal{N}_{S}\left(\begin{array}{c}
I_{0,0} \\
0
\end{array}\right), \\
\mathcal{N}_{A} & =\sqrt{\frac{\omega}{\pi}}\left|g^{0}+\right| p_{z}-\frac{\omega}{2}||^{1 / 2}\left(\frac{1+\omega / g^{0}}{1-\omega / g^{0}}\right)^{1 / 4} \approx \sqrt{\frac{\omega}{\pi}\left|g^{0}+\right| p_{z}||}, \\
\mathcal{N}_{S} & =\sqrt{\frac{\omega}{\pi}}\left|p_{z}+\frac{\omega}{2}\right|^{1 / 2}\left(\frac{1-\omega / g^{0}}{1+\omega / g^{0}}\right)^{1 / 4} \approx \sqrt{\frac{\omega}{\pi}\left|p_{z}\right|},
\end{aligned}
$$

where we also assume that $\omega \ll g^{0}$.

It should be noted that the solutions presented in eqs. (3.21)-(3.26) satisfy the normalization condition,

$$
\int \psi_{N, s, p_{z}}^{\dagger}(x) \psi_{N^{\prime}, s^{\prime}, p_{z}^{\prime}}(x) \sqrt{-g} \mathrm{~d}^{3} x=\delta_{N N^{\prime}} \delta_{s s^{\prime}} \delta\left(p_{z}-p_{z}^{\prime}\right) .
$$


Here $\psi$ and $\psi_{r}$ are related by eq. (3.8).

Using eq. (3.14) we can get the corrections to the energy levels due to the nonzero mass, $E_{A, S} \rightarrow E_{A, S}+E_{A, S}^{(1)}$. On the basis of eqs. (3.21)-(3.25) one finds the expression for $E_{A, S}^{(1)}$ in the limit $\omega \ll g^{0}$,

$$
E_{A}^{(1)}=\frac{m^{2}}{2\left(E_{A}-2 N \omega-g^{0}\right)}, \quad E_{S}^{(1)}=\frac{m^{2}}{2\left(E_{S}-2 N \omega\right)},
$$

Note that eq. (3.28) can be obtained if we retain $m^{2}$ in the operator of eq. (3.14).

If we discuss neutrinos moving along the rotation axis, then $2 N \omega \ll\left|p_{z}\right|$. Using eq. (3.20) we get the energy levels of active neutrinos in this case

$$
E_{A}=\left|p_{z}\right|+g^{0}\left(1+\frac{2 N \omega}{\left|p_{z}\right|}\right)+2 N \omega+\frac{2(N \omega)^{2}}{\left|p_{z}\right|}+\frac{m^{2}}{2\left|p_{z}\right|}
$$

where we also keep the mass correction in eq. (3.28). One can see in eq. (3.29) that $\left|p_{z}\right|+$ $g^{0}+\frac{m^{2}}{2\left|p_{z}\right|}$ corresponds to the energy of a left-handed neutrino interacting with background matter in a flat space-time. The rest of the terms in eq. (3.29) are the corrections due to the matter rotation.

\section{Flavor oscillations of Dirac neutrinos in rotating matter}

In this section we study the evolution of the system of massive mixed neutrinos in rotating matter. We formulate the initial condition for this system and derive the effective Schrödinger equation which governs neutrino flavor oscillations. Then we find the correction to the resonance condition owing to the matter rotation and estimate its value for a millisecond pulsar.

We can generalize the results of section 3 to include different neutrino eigenstates. The interaction of neutrino mass eigenstates with background matter is nondiagonal, cf. eq. (2.7). Therefore the generalization of eq. (3.7) for several mass eigenstates $\psi_{i}$ reads

$$
\left[\mathcal{D}-m_{i}\right] \psi_{i}=\frac{1}{2} \gamma^{\overline{0}} g_{i}^{0}\left(1-\gamma^{\overline{5}}\right) \psi_{i}+\frac{1}{2} \gamma^{\overline{0}} \sum_{j \neq i} g_{i j}^{0}\left(1-\gamma^{\overline{5}}\right) \psi_{j}
$$

where $g_{i}^{0} \equiv g_{i i}^{0}$ and $g_{i j}^{0}$ are the time components of the matrix $\left(g_{i j}^{\mu}\right)$ given in eq. (2.8), $m_{i}$ is the mass of $\psi_{i}$, and $\mathcal{D}$ can be found in eq. (3.7). As in section 3, we omitted the term $(\omega r)^{2} \ll 1$ in eq. (4.1). Note that eq. (4.1) is a generalization of eq. (2.9) for a system of the neutrino mass eigenstates moving in a rotating frame.

We shall study the evolution of active ultrarelativistic neutrinos and neglect neutrinoantineutrino transitions. In this case we can restrict ourselves to the analysis of two component spinors. The general solution of eq. (4.1) has the form,

$$
\eta_{i}(x)=\sum_{N, s} \int \frac{\mathrm{d} p_{z}}{\sqrt{2 \pi}} a_{N, s, p_{z}}^{(i)} e^{\mathrm{i} p_{z} z+\mathrm{i} J_{z} \phi} u_{N, s, p_{z}}(r) e^{-\mathrm{i} E_{i} t},
$$


where

$$
u_{N, s, p_{z}}=\sqrt{\frac{\left|p_{z}\right| \omega}{\pi}}\left\{\begin{array}{cc}
\left(\begin{array}{c}
0 \\
I_{N-1, s}\left(\omega\left|p_{z}\right| r^{2}\right)
\end{array}\right), & \text { if } p_{z}>0, \\
\left(\begin{array}{c}
-\mathrm{i} I_{N, s}\left(\omega\left|p_{z}\right| r^{2}\right) \\
0
\end{array}\right), & \text { if } p_{z}<0 .
\end{array}\right.
$$

The energy levels $E_{i}$ are given in eq. (3.29) with $m \rightarrow m_{i}$. Here we omit the subscript $A$ in order not to encumber the notation. Our goal is to find the coefficient $a_{N, s, p_{z}}^{(i)}=a_{N, s, p_{z}}^{(i)}(t)$. In eqs. (4.2) and (4.3) we neglect the small ratio $\omega / g_{i}^{0}$.

Although in general case the dynamics of a Dirac field obeys the quantum field theory, is some situations, related to the description of neutrino oscillations, we can use first quantized spinors (for the detailed analysis see ref. [19] and references therein). Thus we will suppose that the quantity $a_{N, s, p_{z}}^{(i)}$ in eq. (4.2) is a $c$-number coefficient rather than an operator.

In the following we shall discuss the case of two neutrino mass eigenstates, i.e. $i=1,2$, interacting with rotating background matter. In this situation the mixing matrix $\left(U_{\alpha i}\right)$ in eq. (2.6) can be parametrized with help of one vacuum mixing angle $\theta$.

To describe the time evolution of the system we should specify the initial condition. We shall fix the initial wave functions in the flavor eigenstates basis. One of the possible choices of the initial wave functions is the following: $\nu_{\alpha}(\mathbf{r}, t=0)=0$ and $\nu_{\beta}(\mathbf{r}, t=0) \sim$ $\exp \left(\mathrm{i} p_{z}^{(0)} z+\mathrm{i} J_{z}^{(0)} \phi\right) \delta_{N, N_{0}}$, where $\alpha \neq \beta$ and $p_{z}^{(0)}, J_{z}^{(0)}=1 / 2-N_{0}+s_{0}, N_{0}$, and $s_{0}$ are the quantum numbers of the initial wave function. In the following we shall omit the index 0 in order not to encumber the notations. We will be interested in the evolution of $\nu_{\alpha}$ in the subsequent moments of time. Supposing that $\nu_{\beta} \equiv \nu_{e}$ and $\nu_{\alpha} \equiv \nu_{\mu}$ or $\nu_{\tau}$, we get that the adopted initial condition corresponds to a typical situation of oscillations of neutrinos emitted at the initial stages of a pulsar evolution: one looks for $\nu_{\mu}$ or $\nu_{\tau}$ in a neutrino beam, initially consisting of $\nu_{e}$ only.

Using the fact that $u_{N, s, p_{z}}^{\dagger} \sigma_{2} u_{N, s, p_{z}}=0$, where $u_{N, s, p_{z}}$ is given in eq. (4.3), on the basis of eq. (4.1) we get the effective Schrödinger equation for $\tilde{\Psi}^{\mathrm{T}}=\left(a_{1}, a_{2}\right)$,

$$
\mathrm{i} \frac{\mathrm{d} \tilde{\Psi}}{\mathrm{d} t}=\left(\begin{array}{cc}
0 & g_{12}^{0} \exp \left[\mathrm{i}\left(E_{1}-E_{2}\right) t\right] \\
g_{12}^{0} \exp \left[\mathrm{i}\left(E_{2}-E_{1}\right) t\right] & 0
\end{array}\right) \tilde{\Psi} .
$$

Here we omitted all the indexes of $a_{i}$ besides $i=1,2$. It is convenient to introduce the modified effective wave function $\Psi=\mathcal{U}_{3} \tilde{\Psi}$, where $\mathcal{U}_{3}=\operatorname{diag}\left(e^{\mathrm{i} \Omega t / 2}, e^{-\mathrm{i} \Omega t / 2}\right), \Omega=E_{1}-E_{2}$. Using eq. (4.4), we get for $\Psi$

$$
\mathrm{i} \frac{\mathrm{d} \Psi}{\mathrm{d} t}=\left(\begin{array}{cc}
\Omega / 2 & g_{12}^{0} \\
g_{12}^{0} & -\Omega / 2
\end{array}\right) \Psi .
$$

Note that eq. (4.5) has the form of the effective Schrödinger equation one typically deals with in the study of neutrino flavor oscillations in background matter.

If the transition probability for $\nu_{\alpha} \leftrightarrow \nu_{\beta}$ is close to one, i.e. $P_{\nu_{\beta} \rightarrow \nu_{\alpha}}=\left|\left\langle\nu_{\alpha}(t) \mid \nu_{\beta}(0)\right\rangle\right|^{2} \approx$ 1, flavor oscillations of neutrinos are said to be at resonance. Using eqs. (2.6), (3.1), (3.29), 
and (4.5), the resonance condition can be written as,

$$
\left(f_{\alpha}^{0}-f_{\beta}^{0}\right)\left(1+\frac{2 N \omega}{\left|p_{z}\right|}\right)+\frac{\Delta m^{2}}{2\left|p_{z}\right|} \cos 2 \theta=0
$$

where $\Delta m^{2}=m_{1}^{2}-m_{2}^{2}$ is the mass squared difference.

Let us consider electroneutral background matter composed of electrons, protons, and neutrons. If we study the $\nu_{e} \rightarrow \nu_{\alpha}$ oscillation channel, where $\alpha=\mu, \tau$, using eq. (2.5), we get that $f_{\nu_{\alpha}}^{0}=-\frac{1}{\sqrt{2}} G_{\mathrm{F}} n_{n}$ and $f_{\nu_{\beta}}^{0} \equiv f_{\nu_{e}}^{0}=\sqrt{2} G_{\mathrm{F}}\left(n_{e}-\frac{1}{2} n_{n}\right)$, where $n_{e}$ and $n_{n}$ are the densities of electrons and neutrons. Using eq. (4.6), we obtain that

$$
\sqrt{2} G_{\mathrm{F}} n_{e}\left(1+\frac{2 N \omega}{\left|p_{z}\right|}\right)=\frac{\Delta m^{2}}{2\left|p_{z}\right|} \cos 2 \theta
$$

At the absence of rotation, $\omega=0$, eq. (4.7) is equivalent to the Mikheyev-SmirnovWolfenstein resonance condition in background matter [2].

Let us evaluate the contribution of the matter rotation to the resonance condition in eq. (4.7) for a neutrino emitted inside a rotating pulsar. We make a natural assumption that for a corotating observer neutrinos are emitted in a spherically symmetric way from a neutrinosphere. That is we should take that $l \approx 0$ and $N \approx s$. Then the trajectory of a neutrino is deflected because of the noninertial effects and the interaction with background matter. The radius $\mathcal{R}$ of the trajectory can be found from

$$
\mathcal{R}^{2}=2\left|p_{z}\right| \omega \int_{0}^{\infty} r^{2}\left|u_{N, s, p_{z}}(r)\right|^{2} r \mathrm{~d} r \approx \frac{2 N}{\left|p_{z}\right| \omega},
$$

where we take into account that $N \gg 1$.

We shall assume that $\mathcal{R} \sim R_{0}$, where $R_{0}=10 \mathrm{~km}$ is the pulsar radius. In this case neutrinos escape a pulsar. Taking that $\omega=10^{3} \mathrm{~s}^{-1}$ and using eq. (4.8), we get that the correction to the resonance condition in eq. (4.7) is $\frac{2 N \omega}{\left|p_{z}\right|} \approx\left(R_{0} \omega\right)^{2} \approx 10^{-3}$. The obtained correction to the effective number density is small but nonzero. This result corrects our previous statement in ref. [20] that a matter rotation does not contribute neutrino flavor oscillations.

\section{Summary and discussion}

In summary we notice that we have studied the evolution of massive mixed neutrinos in nonuniformly moving background matter. The interaction of neutrinos with background fermions is described in frames of the Fermi theory (see section 2). A particular case of the matter rotating with a constant angular velocity has been studied in section 3.1. We have derived the Dirac equation for a weakly interacting neutrino in a rotating frame and found its solution in case of ultrarelativistic neutrinos, cf. eqs. (3.21)-(3.23). The energy spectrum obtained in eqs. (3.20) and (3.28) includes the correction owing to the nonzero neutrino mass.

We have used the Dirac equation in a noninertial frame, cf. eq. (3.2), as a main tool for the study of the neutrino motion in matter moving with an acceleration. To develop 
the quantum mechanical description of such a neutrino we have chosen a noninertial frame where matter is at rest. In this frame the effective potential of the neutrino-matter interaction is well defined. However, the wave equation for a neutrino turns out to be more complicated since one has to deal with noninertial effects.

Previously neutrinos weakly interacting with a rotating matter were considered in ref. [21]. To describe the neutrino-matter interaction the authors of ref. [21] used the effective potential in eq. (2.2) with a nonzero velocity of background fermions $\mathbf{u}_{f}$ corresponding to a rotation. It should be, however, noted that the formalism for the study of the neutrino interaction with moving matter derived in ref. [22] is valid when matter has a constant velocity. That is why, despite the similarity of the neutrino wave functions, cf. eqs. (3.22) and (28) in ref. [21] (they are both expressed via Laguerre functions), there is a difference in the neutrino energy spectrum, cf. eqs. (3.20) and (31) in ref. [21].

One can suggest the following explanation of the energy levels discrepancy. It was assumed in ref. [21] that a neutrino is initially created in an inertial frame and then starts to interact with a rotating matter. This situation can be implemented if one studies a neutrino emission in a very center of a rotating pulsar where the matter velocity is negligible. However, as known, in case of a pulsar, neutrinos are typically emitted from a neutrinosphere, which has a significant radius especially for a young and hot star. The surface of the neutrinosphere already has a nonzero velocity. Therefore the description of neutrinos in a rotating matter proposed in ref. [21] is not applicable in this case. In our approach, the quantum numbers $\left(N, s, p_{z}\right)$, characterizing a neutrino state, are measured by a corotating observer. Thus our description can be used even when a neutrino is created in a rotating matter, with all the noninertial effects being accounted for exactly.

Nevertheless one can check that the energy spectrum in eq. (3.20) coincides with that found in ref. [21] for $N \omega \ll p_{z}$. It happens when a pulsar rotates slowly or neutrinos are emitted close the rotation axis, with initial momenta directed along it. In the case we get from eq. (3.20) that $E_{A}-2 N \omega \approx g^{0}+\sqrt{p_{z}^{2}+4 N \omega g^{0}}$, where we keep the term linear in $N \omega$ inside the square root. One can see that, besides the additive term $2 N \omega$, which is due to the noninertial effects, the obtained expression coincides with that derived in ref. [21].

In section 4 we have generalized our results to include various neutrino generations as well as mixing between them. We have derived the effective Schrödinger equation which governs neutrino flavor oscillations. We have obtained the correction to the resonance condition in background matter owing to the matter rotation. Studying neutrino oscillations in a millisecond pulsar, we have obtained that the effective number density changes by $0.1 \%$.

Despite the obtained correction is small, we may suggest that our results can have some implication to the explanation of great peculiar velocities of pulsars. It was suggested in ref. [23] that an asymmetry in neutrino oscillations in a magnetized pulsar can explain a great peculiar velocity of the compact star. An evidence for the alignment of the rotation and velocity vectors of pulsars was reported in ref. [24]. Therefore we may suggest that neutrino flavor oscillations in a rapidly rotating pulsar can contribute to its peculiar velocity. It should be noted that neutrino spin-flavor oscillations, including noninertial effects, in a rapidly rotating magnetized star were studied in ref. [25] in the context of the explanation of high peculiar velocities of pulsars. 


\section{Acknowledgments}

I am thankful to S. P. Gavrilov for helpful comments, to FAPESP (Brazil) for a grant, to Tomsk State University Competitiveness Improvement Program for a partial support, and to Y. S. Kivshar for the hospitality at ANU where a part of the work was made.

Open Access. This article is distributed under the terms of the Creative Commons Attribution License (CC-BY 4.0), which permits any use, distribution and reproduction in any medium, provided the original author(s) and source are credited.

\section{References}

[1] G. Bellini, L. Ludhova, G. Ranucci and F.L. Villante, Neutrino oscillations, Adv. High Energy Phys. 2014 (2014) 191960 [arXiv: 1310.7858] [INSPIRE].

[2] M. Blennow and A.Y. Smirnov, Neutrino propagation in matter, Adv. High Energy Phys. 2013 (2013) 972485 [arXiv: 1306.2903] [INSPIRE].

[3] C. Broggini, C. Giunti and A. Studenikin, Electromagnetic Properties of Neutrinos, Adv. High Energy Phys. 2012 (2012) 459526 [arXiv: 1207.3980] [INSPIRE].

[4] D.V. Ahluwalia and C. Burgard, Gravitationally induced quantum mechanical phases and neutrino oscillations in astrophysical environments, Gen. Rel. Grav. 28 (1996) 1161 [gr-qc/9603008] [INSPIRE].

[5] G. Lambiase, Neutrino oscillations in non-inertial frames and the violation of the equivalence principle. Neutrino mixing induced by the equivalence principle violation, Eur. Phys. J. C 19 (2001) 553 [INSPIRE].

[6] M. Dvornikov and C. Dib, Spin-down of neutron stars by neutrino emission, Phys. Rev. D 82 (2010) 043006 [arXiv:0907.1445] [INSPIRE].

[7] B. Basu and D. Chowdhury, Inertial effect on spin orbit coupling and spin transport, Annals Phys. 335 (2013) 47 [arXiv:1302.1063] [INSPIRE].

[8] C. Giunti and C.W. Kim, Fundamentals of Neutrino Physics and Astrophysics, Oxdord University Press, Oxford U.K. (2007) pgs. 137-179.

[9] M. Dvornikov and A. Studenikin, Neutrino spin evolution in presence of general external fields, JHEP 09 (2002) 016 [hep-ph/0202113] [INSPIRE].

[10] X. Qian and W. Wang, Reactor neutrino experiments: $\theta_{13}$ and beyond, Mod. Phys. Lett. A 29 (2014) 1430016 [arXiv:1405.7217] [INSPIRE].

[11] S.T. Petcov, The Nature of Massive Neutrinos, Adv. High Energy Phys. 2013 (2013) 852987 [arXiv: 1303.5819] [INSPIRE].

[12] A.A. Grib, S.G. Mamaev and V.M. Mostepanenko, Quantum Effects in Intense External Fields: Methods and Results not Related to the Perturbation Theory, Atomizdat, Moscow Russia (1980), pgs. 13-15.

[13] D. Píriz, M. Roy and J. Wudka, Neutrino oscillations in strong gravitational fields, Phys. Rev. D 54 (1996) 1587 [hep-ph/9604403] [INSPIRE].

[14] L.D. Landau and E.M. Lifshitz, The Classical Theory of Fields, Butterworth Heinemann, Amsterdam (1994), 4th edn., pgs. 329-330. 
[15] K. Bakke, Rotating effects on the Dirac oscillator in the cosmic string spacetime, Gen. Rel. Grav. 45 (2013) 1847 [arXiv: 1307.2847] [INSPIRE].

[16] P. Schluter, K. Wietschorke and W. Greiner, The Dirac equation on orthogonal coordinate systems: I. The local representation, J. Phys. A 16 (1983) 1999 [InSPIRE].

[17] C. Itzykson and J.-B. Zuber, Quantum Field Theory, McGraw-Hill, New York U.S.A. (1980), pgs. 691-696.

[18] F.W. Hehl and W.-T. Ni, Inertial effects of a Dirac particle, Phys. Rev. D 42 (1990) 2045 [INSPIRE].

[19] M. Dvornikov, Field theory description of neutrino oscillations, in Neutrinos: Properties, Sources and Detection, J.P. Greene eds., Nova Science Publishers, New York (2011), pgs. 23-90 [arXiv: 1011.4300] [INSPIRE].

[20] M. Dvornikov, Neutrino Flavor Oscillations in Rotating Matter, Azerbaij. Astron. J. 6 (2011) 5 [arXiv: 1001.2516] [INSPIRE].

[21] I. Balantsev, Y. Popov and A. Studenikin, Neutrino magnetic moment and neutrino energy quantization in rotating media, Nuovo Cim. C32N5-6 (2009) 53 [arXiv:0906.2391] [INSPIRE].

[22] A.E. Lobanov and A.I. Studenikin, Neutrino oscillations in moving and polarized matter under the influence of electromagnetic fields, Phys. Lett. B 515 (2001) 94 [hep-ph/0106101] [INSPIRE].

[23] A. Kusenko and G. Segrè, Velocities of pulsars and neutrino oscillations, Phys. Rev. Lett. 77 (1996) 4872 [hep-ph/9606428] [INSPIRE].

[24] S. Johnston et al., Evidence for alignment of the rotation and velocity vectors in pulsars, Mon. Not. Roy. Astron. Soc. 364 (2005) 1397 [astro-ph/0510260] [INSPIRE].

[25] G. Lambiase, Pulsar kicks induced by spin flavor oscillations of neutrinos in gravitational fields, Mon. Not. Roy. Astron. Soc. 362 (2005) 867 [astro-ph/0411242] [INSPIRE]. 